\title{
Femoral Shaft Fracture Occurring as a Result of Physical Manipulation: A Case Report
}

\author{
Rami A. Ayoubi Dany K. Aouad Mohammad M. Darwish Georgio \\ G. Lati Jamal Saade Alexandre H. Nehme \\ Department of Orthopedic Surgery, Saint George Hospital Medical Center, University of \\ Balamand, Beirut, Lebanon
}

\section{Keywords}

Physical manipulative treatment - Femoral fractures - Case report - Osteopathy - Chiropractic . Complication

\begin{abstract}
Treatments including physical manipulation maneuvers have been widely used for the management of various pain complaints. These maneuvers, although generally considered safe, can be associated with serious complications. We report the case of a 37-years-old previously healthy male patient, who presented to our emergency department (ED) for severe right thigh pain and inability to bear weight that developed during a chiropractic session. Imaging done at the ED showed a displaced distal femoral shaft fracture. The majority of the complications of manipulation maneuvers can be as simple as sprains, however, can be drastic or life-threatening in certain cases causing cerebrovascular accidents or major orthopedic fractures. Such complications are rare and definitely underreported. A femoral shaft fracture occurring as a result of physical manipulation has not been previously reported in the literature. We report a distal femoral shaft fracture that was induced by forceful chiropractic manipulation. It is the first to be reported in the literature. This complication should be noted, reported, and the necessary precautions are taken to avoid such major adverse events.
\end{abstract}

(C) 2021 The Author(s).

Published by S. Karger AG, Basel

\section{Introduction}

Many patients refer to chiropractors, osteopathic physicians, or physiotherapists for noninvasive modalities of pain management, especially in the management of nonspecific muscular pain of the neck and the lower back [1]. Knowing that these treatments include 
physical maneuvers that can be forceful, it is necessary that they are performed on selected patients who are not at risk of injuries. In addition, the practitioner should have competent skills. Otherwise, these treatments can be inadequately performed resulting in dangerous outcomes [2].

Vick et al. [3] listed various complications in a retrospective study, which included bone fractures, dislocations, disc herniations, and even cerebrovascular accidents. These complications were reported after chiropractic sessions which included force and high-velocity maneuvers [3].

There are no previous cases in the literature reporting femoral shaft fractures resulting from physical manipulation. This report describes the case of a 37-years-old healthy male patient who was found to have a transverse fracture of the distal femoral shaft that occurred during a chiropractic session.

\section{Case Presentation}

Written informed consent was obtained from the patient for publication of this case report and any accompanying images. This is a 37-years-old healthy male patient, with no history of chronic medications use nor any physically enhancing drugs, admitted to our emergency department (ED) for the management of severe right thigh pain and inability to ambulate which occurred during a chiropractic session done immediately prior to presentation, before which the patient did not complain of any similar pain or previous history of trauma or fractures.

As per the patient, he visited his chiropractor after a 2-week history of low back pain with right-sided radiculopathy without consulting any spine specialist. During the chiropractic session, and after spinal maneuvers, forceful repetitive flexion and valgus maneuvering were applied to the right lower limb that resulted in a popping sensation with sudden onset of severe right distal thigh pain with an inability to bear weight. He was transferred by his chiropractor to our ED for proper investigations and management of his condition. On physical exam, the patient had severe tenderness on palpation of the distal thigh; swelling was also identified with intact neurovascular status of the right lower limb. Patient was unwilling to flex or extend his knee due to severe pain. Knee X-rays were ordered and showed a right displaced distal third transverse femoral shaft fracture (Fig. 1, 2). Hence, a decision to proceed with urgent surgical treatment of his fracture by an intramedullary nail was taken.

However, due to financial problems related to third-party payers, the patient was unable to undergo the procedure at our university hospital and was transferred to a public hospital for surgical management of his femoral shaft fracture. Attempts to contact the patient for follow-up were unsuccessful as the telephone number registered in the ED patient's file was unreachable.

\section{Discussion}

Several case reports, case series, and systematic reviews describing physical manipulation-induced injuries exist in the literature [2]. The frequency of such injuries is not definite and varies between different studies. Under-reporting of such complications may definitely mask their prevalence. Frequency varies from 1.3 cases per 100,000 and can be as low as 0.05-1.46 per 10,000,000 [4,5]. Physical manipulation can be a safe noninvasive modality of pain management, however, serious complications such as spine fractures, radiculopathies, myelopathies, and cerebrovascular events have been described [6].

\section{Karger'}


Fig. 1. Right knee anteroposterior view X-ray showing a transverse distal third femoral shaft fracture with lateral displacement of the distal fragment.

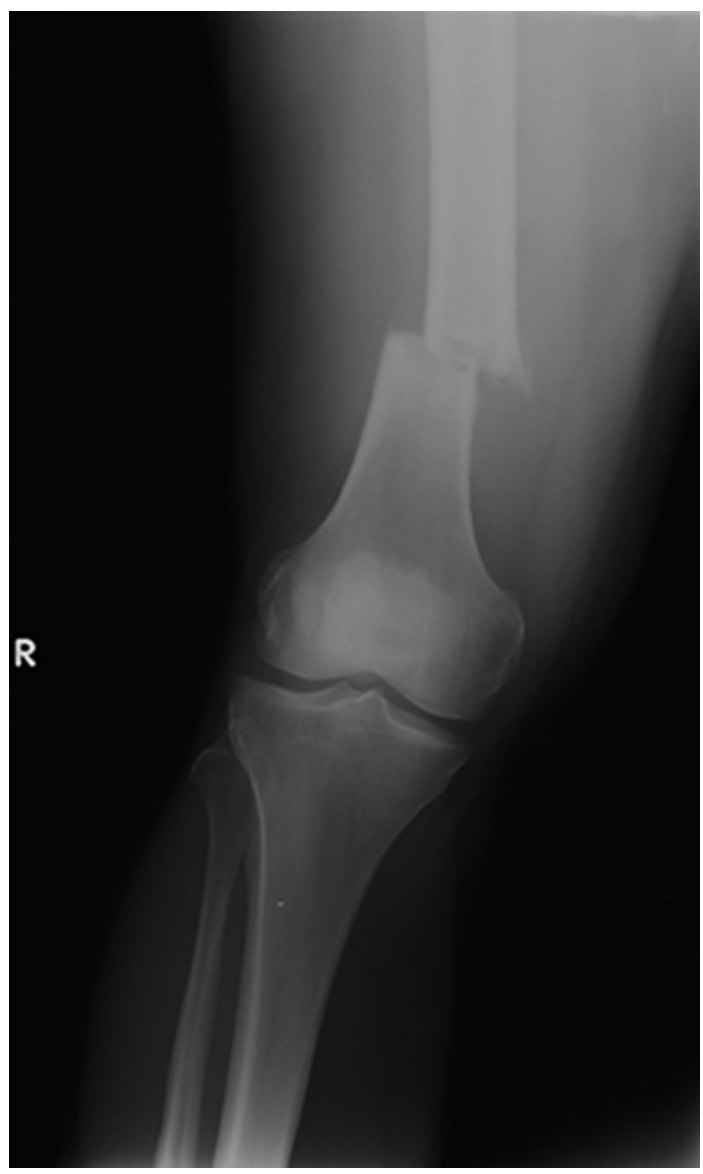

In a review by Vick et al. [3], these complications were classified as minor and major. The minor complications being sprains and rib fractures, and the major ones being cerebrovascular accidents such as vertebral artery dissection in cervical manipulation, major bone fracture dislocations, and disc disease [3].

Injuries to the brainstem and spinal cord, and cauda equina that have caused death in extreme cases were reported as chiropractic complications by Rinsky et al. [7, 8]. Basilar artery thrombosis can result from neck manipulation leading to infarction of the brainstem and death in a significant percentage of cases [7].

Vertebral artery dissection after a chiropractor neck manipulation has been reported in a middle-aged schoolteacher, requiring treatment with mannitol and a ventriculostomy followed by a satisfactory functional recovery [9]. Patients who have a history of coagulation defects, aortic dissection or aneurysms, osteoporosis, and use of anticoagulation therapies should be handled with caution by practitioners involved in physical manipulation, bearing in mind the discussed deadly complications that can take place [2].

The rate of complications due to physical manipulation is considered to be low $[4,5]$. However, these complications can be serious and can lead to death [7, 8]. Furthermore, there exists no solid data about the prevalence of these complications which makes further studies crucial for a better evaluation of their risk and frequency [5].

Unfortunately, our patient was transferred to another hospital for surgical management of his condition. This is a weakness of this article. However, the main purpose of reporting this case is to document and spread awareness of the possible serious complications of physical manipulation of the extremities. 
Case Reports in Orthopedic Research
Case Rep Orthop Res 2021;4:84-88

DOI: $10.1159 / 000515565$

(c) 2021 The Author(s). Published by S. Karger AG, Basel www.karger.com/cio

Ayoubi et al.: Femoral Shaft Fracture Induced by Physical Manipulative Therapy

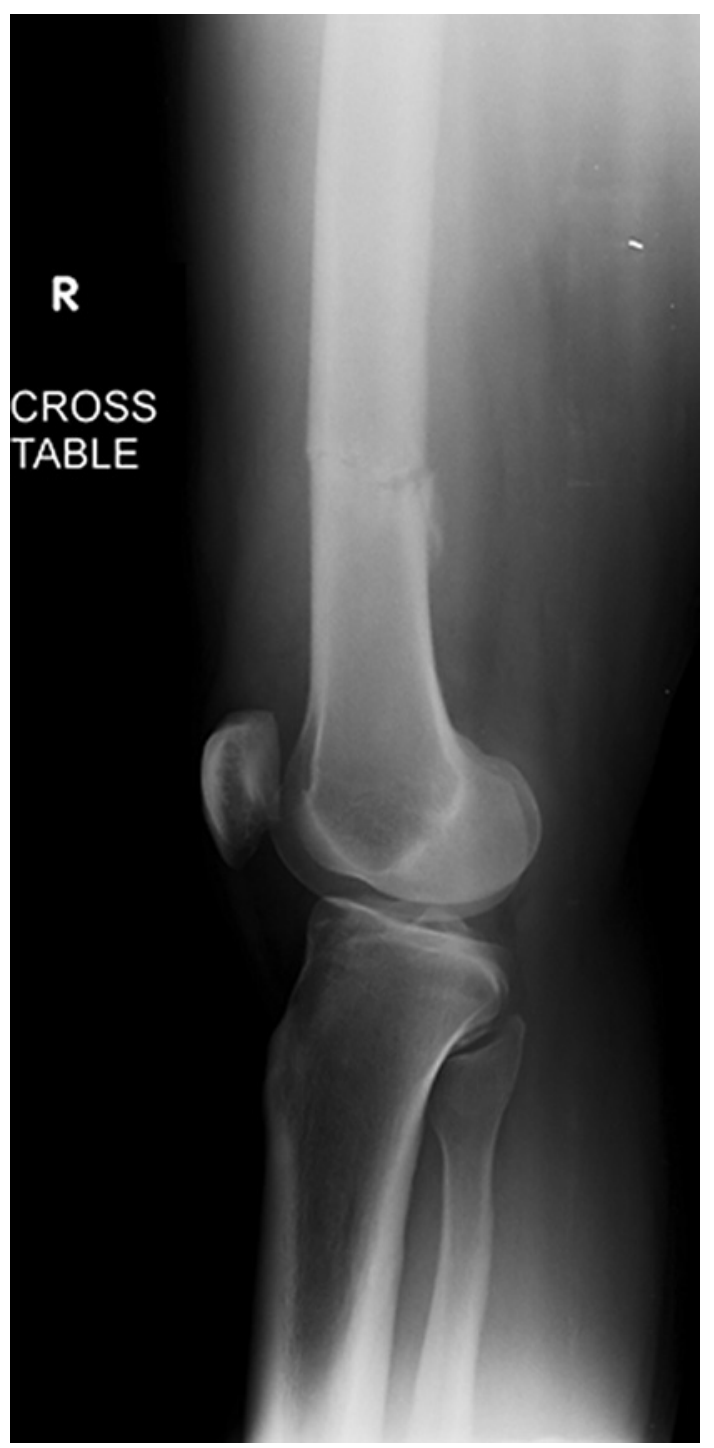

Fig. 2. Right knee lateral view X-ray showing a transverse distal third femoral shaft fracture.

There have been no previous publications on femoral shaft fractures resulting from osteopathic or chiropractic manipulation. There has been only one previous case report of a femoral neck fracture in an elderly patient sustained as a result of physical manipulation [10].

Therefore, this is a case report that should be noted. These complications may seem rare; however, under-reporting of such occurrences may mask its prevalence.

\section{Conclusion}

Using forceful maneuvers in modalities like chiropractic management and osteopathic medicine is not always complication-free. These treatments can be associated with mild to extremely serious complications including death. Primary care physicians and chiropractors should be aware of these complications and rule them out in patients who present after such treatment modalities when suspected. 
Case Reports

in Orthopedic

Research
Case Rep Orthop Res 2021;4:84-88

\begin{tabular}{l|l}
\hline DOI: 10.1159/000515565 & @ 2021 The Author(s). Published by S. Karger AG, Basel \\
\hline
\end{tabular} www.karger.com/cio

Ayoubi et al.: Femoral Shaft Fracture Induced by Physical Manipulative Therapy

\section{Statement of Ethics}

Approval and consent of the Ethics Committee have been received for the publication of this article. Written informed consent was obtained from the patient for publication of this case report AND any accompanying images.

\section{Conflict of Interest Statement}

The authors declare that they have no competing interests

\section{Funding Sources}

No funds were received in support of this study.

\section{Author Contributions}

R.A.: writing and editing the article. M.D.: writing and editing the article. D.A. writing and editing the article, with editing of the images and radiographs: writing and reviewing the article along with the referencing: writing and finalizing the article. A.N.: surgeon who supervised writing and finalizing the article.

\section{Data Availability Statement}

The datasets used and/or analyzed during the current study are available from the corresponding author on reasonable request.

\section{References}

1 Stevinson C, Ernst E. Risks associated with spinal manipulation. Am J Med. 2002;112(7):566-71.

2 Whedon JM, Mackenzie TA, Phillips RB, Lurie JD. Risk of traumatic injury associated with chiropractic spinal manipulation in medicare part B beneficiaries aged 66 to 99 years. Spine. 2015;40(4):264-70.

3 Vick DA, Mckay C, Zengerle CR. The safety of manipulative treatment: review of the literature from 1925 to 1993. J Am Osteopath Assoc. 1996 Jan;96(2):113.

4 Rothwell DM, Bondy SJ, Williams JI. Chiropractic manipulation and stroke: a population-based case-control study. Stroke. 2001;32(5):1054-60.

5 Gouveia LO, Castanho P, Ferreira JJ. Safety of chiropractic interventions: a systematic review. Spine. 2009; 34(11):E405-13.

6 Liao C-C, Chen L-R. Anterior and posterior fixation of a cervical fracture induced by chiropractic spinal manipulation in ankylosing spondylitis: a case report. J Trauma. 2007;63(4):E90-4.

7 Rinsky LA, Reynolds GG, Jameson RM, Hamilton RD. A cervical spinal cord injury following chiropractic manipulation. Paraplegia. 1976;13(4):223-7.

8 Pratt-Thomas HR, Berger KE. Cerebellar and spinal injuries after chiropractic manipulation. J Am Med Assoc. 1947 Jan;133(9):600.

9 Jones J, Jones C, Nugent K. Vertebral artery dissection after a chiropractor neck manipulation. Proc Bayl Univ Med Cent. 2015;28(1):88-90.

10 Ayoubi RA, Nassour NS, Saidy EG, Aouad DK, Maalouly JS, Daou EB. Physical manipulation-induced femoral neck fracture: a case report. Case Rep Orthop Res. 2020;3:118-22. 